\title{
Herbert Kitschelt, Kirk A. Hawkins, Juan Pablo Luna, Guillermo Rosas y Elizabeth J. Zechmeister, Latin American Party Systems (Sistemas de Partidos Latinoamericanos), Cambridgre University Press, Cambridge, 2010.
}

\section{GERMÁN BIDEGAIN PONTE}

Pontificia Universidad Católica de Chile

\section{ROODY RESERVE}

Pontificia Universidad Católica de Chile

Latin American Party Systems es pariente directo de una ola de literatura politológica de mediados de la década de los 90 que examina la calidad de las democracias en América Latina. Se inserta en la tradición inaugurada por Mainwaring y Scully (1995), ${ }^{1}$ de vincular el estudio de los sistemas de partidos con el desempeño de las democracias.

Comencemos por destacar algunos puntos fundamentales de la obra en consideración. En primer lugar, a diferencia de Mainwaring y Scully (1995), el foco no está puesto en la institucionalización per se, sino en un tipo específico de vinculación partidos-votantes: el vínculo programático. Los sistemas de partidos programáticamente estructurados (de ahora en adelante PPS, por su sigla en inglés) serían los únicos capaces de instaurar democracias plenas. En segundo lugar, se usa un argumento estructuralista para explicar el mecanismo de instauración del vínculo programático. Se privilegian causas de largo plazo en detrimento del potencial transformador de alteraciones institucionales y económicas en el corto plazo. Esto marca un cambio en el enfoque dominante, el institucional, de los estudios de los sistemas de partidos latinoamericanos. En tercer lugar, el libro tiene una fuerte pretensión teórica. El argumento sobre el vínculo programático partidos-votantes y sus efectos en las relaciones de accountability democrático busca constituirse en una explicación que pueda viajar en el espacio y en el tiempo para explicar los vínculos partidos-votantes en otros sistemas políticos.

El interés de Kitschelt et al. en un tipo específico de vinculación entre partidos y votantes, el programático, es justificado por dos razones. En primer lugar, la existencia de enfoques normativos que suponen que el modelo de gobernanza democrática de partido responsable es el modelo más legítimo de organización democrática (p. 21). En segunda instancia, y en relación a otros tipos de vinculaciones, la conexión programática suele tener, en promedio, mejores capacidades para mantener en buen estado al funcionamiento de las democracias (p. 29, y capítulo 9). Por lo tanto, y a pesar de que el título del libro es bastante genérico, la motivación de los autores es entender cómo se llega a establecer un tipo específico de vinculación partidovotantes, el PPS. Esto teniendo en cuenta, además, que suele ser más costoso generar PPS que vinculaciones de otro tipo (clientelares, carismáticas, etc.).

El argumento central del libro es el siguiente. La generación de vínculos programáticos en los sistemas de partidos requiere de un aprendizaje relativamente lento, acumulado en capas sucesivas, y caracterizado por ensayo y error. Aun cuando las variables de corto plazo pueden tener una importante influencia, por sí solas no pueden explicar la instalación

1 Ver Mainwaring, Scott y Timothy Scully (1995). "Introduction: Party Systems in Latin America". En Building Democratic Institutions. Party Systems in Latin America, eds. Scott Mainwaring y Timothy Scully, pp. 1-34. Stanford, California: Stanford University Press. 
exitosa de vínculos programáticos (aunque sí la destrucción o erosión de los mismos). Por lo tanto, es necesario tener una mirada de largo plazo para comprender por qué algunos países logran establecer vínculos de este tipo y otros no. Desde la perspectiva teórica del trabajo, la distinción entre causas de largo y corto plazo es fundamental. Concretamente, para que se dé un proceso de aprendizaje y acumulación paulatina que lleve al establecimiento de vínculos partidarios programáticos, un sistema político debe reunir tres elementos fundamentales: capacidades que generen el desarrollo de los actores políticos, oportunidades de organización colectiva y competencia partidaria electoral reiterada, de modo que el electorado y los actores políticos puedan construir vínculos programáticos, y political stakes que puedan dar forma a los alineamientos partidarios.

De acuerdo con su teoría, Kitschelt et al., encuentran que los países latinoamericanos que tienen condiciones más favorables para presentar un desarrollo de PPS son aquellos que experimentaron cierto nivel de desarrollo económico en las décadas previas a la Segunda Guerra Mundial (capacidades), que vivieron largos períodos de competencia democrática después de 1945 (oportunidades) y que implementaron políticas de atención social relativamente extendidas al menos en los sectores urbanos (generando stakes sobre cobertura, mantenimiento, etc., de estas políticas) (pp. 8-9). Esto respecto a la mirada de largo plazo. Con respecto al corto plazo, y de acuerdo con su teoría, también pueden identificarse capacidades, oportunidades y stakes. Tal es el caso de booms o crisis económicas, reformas o crisis institucionales, y la evaluación constante del electorado respecto a la performance de los líderes políticos. Pero la tesis de los autores es que allí donde no existan causas de largo plazo favorables para la generación de una estructuración programática, un boom económico o algún tipo de reforma institucional no lograrán constituirlo. No obstante, en casos donde sí existe un PPS en alguna medida, una fuerte crisis económica o un reverso institucional, pueden erosionar este tipo de vínculo o incluso hacerlo desaparecer (por ejemplo, la crisis económica argentina de 2001-2002).

¿Cómo es conceptualizado el PPS? Esto es fundamental para la teoría, así como para su contrastación empírica. La estructuración programática del sistema de partidos se compondría de dos elementos. En primer lugar, la estructuración programática al nivel de élites (políticos y partidos). En segundo lugar, el vínculo programático entre las élites y los votantes. Estos dos aspectos se encuentran mediados por la coordinación programática que, relacionándolos, hace posible el PPS. Establecido el concepto de PPS y el modelo teórico, los autores se abocan a dos tareas principales: 1) describir y mapear el grado de PPS en América Latina, y 2) desvelar los vínculos causales que determinan los distintos grados de PPS en la región, explicar las consecuencias de estas diferencias para las democracias y especular sobre su desarrollo futuro.

La Parte I del libro aborda la primera tarea a través del estudio de los cuatro indicadores que componen el índice de PPS: i) dimensionalidad del espacio ideológico, ii) semánticas de izquierda-derecha, iii) cohesión ideológica de los partidos políticos y iv) representación sobre issues (cada indicador es abordado en un capítulo específico). Los primeros tres indicadores sirven para estudiar la coordinación entre élites, mientras que el cuarto busca captar los vínculos entre élites y votantes. La consecuencia del establecimiento de vínculos programáticos debería expresarse en a) baja dimensionalidad del espacio ideológico (cada partido debe concentrarse en pocas dimensiones que lo definen), b) una semántica normativa sobre el eje izquierda-derecha (simbólica, pero más que nada sustantiva), c) cierta cohesión ideológica de los partidos y d) una representación adecuada de los intereses del electorado sobre los temas principales. La agregación de estos indicadores compone el índice de PPS propuesto en el trabajo. 
Los capítulos sobre la coordinación entre las élites se basan exclusivamente en evaluar las respuestas de los legisladores a la encuesta de elites cursada, en 1997, por el Programa de Estudio de Elites de América Latina (PELA) de la Universidad de Salamanca. En el capítulo dedicado a los vínculos entre partidos y votantes, se agrega la utilización de Latinobarómetro para el año 1998 (de modo de comparar respuestas de élites y de la opinión pública). Esta es la evidencia empírica fundamental de la parte descriptiva del trabajo, con la que se procura determinar el estado de los sistemas de partidos latinoamericanos hacia 1998. Su utilización requiere de algunos comentarios. En primer lugar, el hecho de trabajar con observaciones para un solo momento específico parece limitar el alcance de los hallazgos. Aunque el diseño cross-sectional permite realizar comparaciones entre casos, veremos más adelante que mirar el estado de los sistemas de partidos en un solo momento puede acarrear dificultades, notoriamente a la hora de establecer la importancia de las causas de corto plazo. Por otra parte, respecto al uso de PELA, no parece evidente que se pueda usar la opinión personal de los legisladores, su autoubicación ideológica, para establecer el posicionamiento de sus partidos y, por otro lado, equiparar actitudes con comportamiento. Sin dudas, aquí hay una opción metodológica trascendental. Los autores son conscientes de las críticas que esto puede suscitar, y debaten distintos puntos de vista y justifican su decisión (pp. 65-67). Será la tarea de futuras investigaciones establecer la justeza de haber tomado tal camino. En todo caso, es una avenida de investigación abierta por el libro cuya ventaja y desventaja futuros estudios tendrán que evaluar.

Por otro lado, dado que el libro tiene pretensiones teóricas amplias, puede objetarse que haga descansar tal propósito en una única observación en el tiempo, de 12 casos (o 9 en el capítulo 4). Un primer atisbo de posibles problemas de este argumento puede encontrarse en unas rápidas observaciones sobre el caso chileno. Mientras que cada página del libro va confirmando la idea de que Chile es el país con mayores puntajes en el vínculo programático de sus partidos con los electores; al mismo tiempo, existe evidencia empírica de otras procedencias que señala clara desafección entre ciudadanos y partidos (LAPOD, 2010 de próxima aparición). Por ejemplo, Chile, comparado con el resto de América Latina, tiene la tasa de participación electoral más baja entre los jóvenes (Toro, 2008). ${ }^{2}$ ¿Pueden compaginarse sistemas de partidos con altos PPS pero desconectados de los ciudadanos? ¿Cómo se explica esta diferencia entre lo vaticinado por la teoría y la realidad chilena?

En la parte II del libro, la tarea principal consiste en presentar explicaciones causales que den cuenta de las observaciones presentadas en la primera parte. Esta empresa es realizada bajo el lente del modelo teórico de la obra, y concretamente a través de la revisión de estudios de casos para cada país (p. 173) e información secundaria sobre los mismos. Así, en el sexto capítulo se trabaja sobre las causas de largo plazo y sus consecuencias sobre los sistemas de partidos estudiados. Grosso modo, se confirma el argumento presentado al comienzo de esta recensión: sólo países con cierto desarrollo económico previo a 1950, con ciertas garantías políticas y competencia electoral durante períodos extendidos de tiempo y con políticas sociales vinculadas al desarrollo de ISI generaron vínculos programáticos en torno a temáticas económico-distributivas. Sin embargo, México y Brasil aparecen como importantes casos desviantes. Mientras que el primero no presenta condiciones propicias para PPS, los resultados sorprenden arrojando cierta presencia de estructuración programática. Brasil es el caso opuesto, a pesar de presentar características que según la teoría deberían desarrollar PPS, sus puntajes son bajos en el índice. Los autores avanzan explicaciones ad hoc para solucionar

2 Toro Maureira, Sergio. (2008). “De lo épico a lo De lo épico a lo cotidiano: Jóvenes y generaciones políticas en Chile", Revista de Ciencia Política, Volumen 28 , Issue: 2, pp. 143-170. 
estas inconsistencias. Acto seguido se abordan las causas de corto plazo y sus posibles consecuencias sobre el tipo de estructuración de los sistemas de partido. En este capítulo se hacen patentes algunas de las limitaciones de trabajar con una sola observación en el tiempo que ya esbozamos. Notoriamente, el no poder observar varianza dentro de los casos debilita el argumento respecto a las causas de corto plazo. Sin observaciones sistemáticas que nos permitan determinar las oscilaciones en el PPS causadas por capacidades u oportunidades de este tipo, parece en cierta medida osado descartar tan tajantemente las capacidades constructivas de vínculos programáticos en plazos de no tan largo aliento. De todos modos, los autores buscan dar sustento con la información disponible a su argumento principal: las causas de corto plazo pueden tener efectos de erosión o destrucción del PPS, pero no de construcción de este tipo de vínculo entre partidos y votantes.

El capítulo 8 se aboca al testeo de la capacidad de la religión y el régimen político para explicar la estructuración de PPS, ya que hasta este entonces el foco central era en la estructuración en torno a temas económico-distributivos. Con respecto a la religión, la conclusión es en cierta medida ambigua. Por un lado, Krlschelt et al. encuentran que la religión puede considerarse como un gatillador importante de movilizaciones que crean stakes políticos a largo plazo, basados en el conflicto estado-religión; pero, por otro lado, no explican la emergencia de partidos religiosos (los Partidos Demócrata Cristianos) en la región. El estudio de régimen político como estructurador de PPS es menos riguroso pues, como señalan los autores, disponen de menos evidencia empírica. Sin embargo, es interesante destacar que encuentran que en los casos en que este corte es más importante, las causas de corto plazo tienen mayor importancia con respecto a la estructuración sobre temas económicos o religiosos (p. 276). El capítulo siguiente aborda las consecuencias del PPS para el funcionamiento de las democracias. Allí se presenta una serie de correlaciones que muestran vínculos entre el grado de PPS y algunos atributos de las democracias que suelen señalarse como deseables (participación y competencia políticas, apoyo a la democracia y calidad de la gobernanza). Estas relaciones son testeadas con hipótesis rivales económicas, y los resultados marcan la preponderancia del PPS. Aunque la evidencia no permite llegar a resultados concluyentes ni establecer fuertes vínculos causales, da luces sobre la vinculación entre PPS y algunos aspectos de la calidad de las democracias. En este sentido, en el capítulo se vuelve y da sustento empírico a las motivaciones que justificaban el interés por este tipo específico de vinculación entre partidos y votantes: aquellos sistemas políticos estructurados programáticamente tienen en términos generales un desempeño democrático mejor que sistemas cuya estructuración programática es menor.

El capítulo 10 que cierra el libro comienza realizando un esfuerzo de testeo de la teoría en casos allende las fronteras latinoamericanas. La conclusión global es que sí se pueden usar los mismos indicadores para explicar la realidad de los ex países comunistas de Europa Central y del Este. Este argumento reforzaría una idea destacada al principio de este comentario, a saber las pretensiones teóricas generales de este libro. Pero, por otro lado, tratándose del caso latinoamericano, el libro termina con unas notas bastante pesimistas sobre el futuro de la región, en términos de capacidad para estructurar y mantener PPS. Los países son más propensos a ser víctimas de shocks económicos externos que destruyen PPS, mientras que los crecimientos económicos de corto plazo no estructuran PPS. Aun cuando se había mantenido la idea que no se ofrecía una visión de path dependence cerrado (p. 9), esta última valoración sobre el futuro de América Latina va en este sentido. De igual manera, se sostiene que los llamados "neosocialismos" y populismos actuales en América Latina son incapaces de generar PPS en el largo plazo. Como puede verse, este argumento tiene cierto cariz ideológico. Si no ¿por qué asumir teóricamente y a priori que los "neosocialismos" no puedan significar a la larga un mecanismo de incorporación de nuevos segmentos sociales, tal como sucedió con la ISI en su momento? 
En síntesis, no obstante las críticas puntuales esbozadas a lo largo de este comentario, Latin American Party Systems es un excelente libro que destaca principalmente por sus aportes teóricoconceptuales y la innovadora manera propuesta de medir PPS. Cumple su objetivo de resaltar la importancia de los tipos de vínculos entre partidos y votantes para el estudio de los sistemas de partidos y las democracias. De hecho, aun cuando su principal preocupación son los vínculos programáticos, puede servir de catalizador para el estudio de otro tipo de vínculos, como los clientelares que abundan en la región. Además, contribuirá a mirar los sistemas de partidos latinoamericanos desde un enfoque novedoso, contrabalanceando el institucionalismo reinante. Sin dudas, es un gran aporte al estudio de la política comparada. Ofrece una nueva mirada de las democracias y sistemas de partidos del continente, y también un marco conceptual que será de mucha utilidad para los estudiosos de los sistemas de partidos, tanto de América Latina como de otras latitudes. 
